\title{
Brain hyperstimulation by epileptic seizures
}

\begin{abstract}
There are several ways of inducing brain hyperstimulation in rats and monkeys to study biochemical cerebral differences between orders, one of which is by convulsive drugs, glutamatergic stimulators or GABAergic inhibitors such as pentylenetetrazol. This work aims to explain the functioning of this drug in the brain that allows hyperstimulation.
\end{abstract}

Keywords: hyperstimulation, epiletic seizures, brain
Volume I Issue $6-2017$

\author{
Vanessa Novaes Barros \\ Department of Physiology, Universidade Estácio de Sá, Brazil
}

Correspondence: Vanessa Novaes Barros, Department of Physiology, Universidade Estácio de Sá, Rod. Mário Covas, Cond. Park Italia, I78 (rua roma, 20), Ananindeua - PA- Cep 670I5000, Brazil,Tel 55-I|-981320619, Email barros.novaes@hotmail.com

Received: November 13, 2017 | Published: December 12, 2017

\section{Mini review}

In 2005, the International League Against Epilepsy (ILAE) defined epilepsy as a disorder of the brain characterized by a predisposition to generalized epileptic seizures with neurobiological, cognitive, psychological, and social consequences. The epileptic seizure would be a temporary occurrence of signs and/or symptoms attributed to abnormal and/or synchronic excessive neuronal activity in specific areas of the central nervous system. ${ }^{1}$ The excitability of an individual nerve cell in the brain depends on a number of factors including: excitatory properties of the membrane and microenvironment, intracellular processes, structural neuronal elements, and interneuronal connections. ${ }^{2}$ Any excitable membrane, including that of the neuron, maintains a potential difference between the intracellular and extracellular medium as a result of the selective permeability of some ions, particularly sodium, potassium and chloride ions. The mutation of the genes responsible for the coding of the proteins that form the channels for the passage of these ions through the membrane can cause congenital epilepsy. For example mutation of KCNQ genes 2 and 3 causes neonatal epilepsy, the LGL1 gene causes lateral temporal lobe epilepsy as well as genes SCN1A, SCN2A, SCN1B and others. The first four genes related to the formation of potassium channels and the last two with the formation of sodium channels. ${ }^{2}$

The resting membrane potential of a nerve cell is around -60 to $-80 \mathrm{mv}$. This potential is maintained thanks to the performance of an ATP-bound protein called the sodium and potassium pump. In addition, passive ionic currents and electrochemical equilibrium also contribute to the formation of membrane potential. Many pharmacological agents alter neuronal excitability using these ionic mechanisms. Fluoracetate, fluorocitrate and methionine sulphoximine promote depolarization of the membrane by disrupting the functioning of the sodium/potassium pump. Perchlorate and thiocyanate cause neuronal excitation by altering the ability of glial cells to capture extracellular ions. $^{2}$

In addition to these membrane aspects, other intracellular processes may also be responsible for epileptic seizures. Changes in proteins with carrier function such as the GLUT1 glucose carrier may result in epileptic-associated encephalopathies due to a mutation of the SLC2A1 gene responsible for GLUT1 protein coding. ${ }^{3}$ Another transporter protein responsible for the elimination of toxins in the brain called P-glycoprotein (PCP) can also cause seizures due to its ability to reduce the action of antiepileptic drugs in the epileptogenic regions. In this sense, it is associated with drug resistance. ${ }^{4}$ The influx of calcium into the cell can also promote the opening of channels in the membrane, release of neurotransmitters in the synaptic cleft as well as is involved in processes of removal or addition of channels in the membrane and is therefore a common cause of neuronal hyperexcitability that can result in crisis epileptic. In addition, it can induce selective genes that encode proteins for specific purposes. As with the use of the seizure drug pentilenotetrazole, which causes the expression of c-Fos causing cell growth, cellular differentiation, learning and memory and under certain conditions, leading to the development of chronic epilepsy. ${ }^{5}$

Pentylenetetrazole, also known as metrazol, pentamethylenetetrazole, cardiazole, here abbreviated as PTZ, acts by binding at recognition sites of picrotoxins and benzodiazepines at receptors, postsynaptic, GABAA. As a result of this binding there is a depression of the action of inhibitory neurotransmitters (mainly GABA) making the system hyperexcitable. ${ }^{6}$ Excess neuronal excitation can corrupt cellular function, osmotic damage results in chloride inflow that brings water into the cell. In these cases, there may be excessive calcium influx which thus not only mediates gene expression but also activates proteases and lipases and can cause cell death. ${ }^{6}$ In the scientific community there are 3 main models with PTZ:1-single administration of convulsive dose; 2- kindling, caused by repeated subvulsive doses over time (usually days) and 3 - application of several low (but convulsive) doses with intervals without crisis. The three models cause short-term epileptic seizures and, initially, they present with absence of neuronal degeneration. Therefore, the use of PTZ is indicated to study mechanisms of early cellular responses, induced by epileptic seizures and that develop, at first, independently of neuronal death ${ }^{6}$. Repeated treatment with PTZ causes structural changes mainly in pyramidal cells and interneurons. Studies with kindling in rats demonstrated a decrease in cholinergic cells as well as losses of some GABAergic receptor subunits. PTZ stimulation induced activation of caspases-3 involved in apoptotic degeneration, but in adult rats this activation only occurs months after induction with PTZ. ${ }^{3}$

Mitochondrial dysfunctions can also be evidenced by the increase in the production of reactive species of oxygen and nitric oxide, a process that precedes neuronal death and epileptogenesis. In addition, mitochondria are involved in calcium homeostasis and in the synthesis 
of neurotransmitters. ${ }^{4}$ Disappearance of dendritic microtubules, breakdown of cytoskeletal elements (among them actin) and decrease in the number of dendritic spines are also structural alterations following PTZ-induced seizures. ${ }^{6}$ Cellular responses of recurrent epileptic seizures through PTZ involve inflammation (vasodilation and perivascular proliferation of leukocytes), neurogenesis and reactive glioses in different animal species, including zebrafish, rodents and primates. However, evidence of epileptogenesis (only after numerous seizures with PTZ) was only found in rodents and primates, probably because of the high regeneration capacity found in zebrafish brain.?

According to Cole et al., ${ }^{8}$ there are numerous biochemical, anatomical and functional consequences of epileptic seizures in the central nervous system. These are:

i. Ionic flow, since epilepsy is characterized by repeated and synchronous depolarization waves of neurons. Neurotransmitters interacting with receptors activate ionic fluxes through inotropic channels, including calcium flux.

ii. Activation of kinases. A few minutes are needed for the activation of mitogenic activating kinases ( $\mathrm{p} 44 / 42-\mathrm{MAP}$ kinase or Erk1/2) responsible for a series of responses to epileptic seizures, including the regulation of neurotransmitter release in the synaptic cleft.

iii. Expression of immediate expression genes (such as c-Fos, FosB, JunD and c-Jun) that are related to processes of differentiation, cell death and cell proliferation.

iv. Expression of late genes encoding peptides, cytokines, glial fibrillary acid proteins (GFAPs) and cytoskeleton proteins.

v. Modification of proteins. It has already been shown that a single epileptic seizure is capable of increasing the synthesis of the peptide-glycoprotein-alpha-amine-monoxygenase enzyme that converts peptide glycoprotein to alpha-amine. ${ }^{8}$

vi. In the hippocampal complex, mossy fibers and synaptic reorganization that extends the supragranular cellular layer.

vii. Cellular loss usually in epileptic seizures induced by pilocarpine, ac. kinic or bicuculina, or after hypoxia-ischemia-induced epilepsy mainly in regions of the limbic system including granular cells, hilar interneurons, CA3, CA1, pyramidal cells, amygdala, hypothalamus, entorhinal cortex, septum, dorsalmedial thalamus and cingulate gyrus . viii. Glioses. Glial activation is defined by increased GFAP expression and is related to neurotransmitter reuptake, catabolism, glucose transport, and trophic support. Therefore this phenomenon may be related to the long-term consequences of epileptic seizures.

ix. Neo-neurogenesis in the hippocampus with the appearance of granular cells in the yarn and granule cell layer are observed a few days after pilocarpine-induced seizures.

$\mathrm{x}$. Increased susceptibility to recurrent seizures.

xi. Memory/learning/behavior deficit related to neuronal loss in the hippocampal regions, mainly. ${ }^{8}$

\section{Acknowledgements}

The author thanks a Estácio de sá University, Universidade Federal de São Paulo and Luiz Eugênio Mello for suport.

\section{Conflict of interest}

The author declares no conflict of interest.

\section{References}

1. Fisher RS, Velasco AL. Electrical brain stimulation for epilepsy. Nature Reviews Neurology. 2014;10:261-270.

2. Engel J. Seizures and epilepsy. 2nd ed. USA: Oxford; 2013.736 p.

3. De Vivo DC, Trifiletti RR, Jacobson R, et al. Defective glucose transport across the blood-brain barrier as a cause of persistent hypoglycorrhachia, seizures and developmental delay. N Engl J Med. 1991;325(10):703-709.

4. Locher W, Schmidt D. New developments in antiepileptic drug resistance: an integrative view. Epilepsy Curr. 2009;9(2):47-52.

5. Wayman GA, Davare M, Ando H, et al. An activity-regulated microRNA controls dendritic plasticity by downregulating p250GAP. Proc Natl Acad Sci USA. 2008;105(26):9093-9098.

6. Zhvania MG, Ksovreli M, Japaridze NJ, et al. Ultrastructural changes to rat hippocampus in pentylenetetrazol and kainic acid-induced status epilepticus: A study using eléctron microscopy. Micron. 2015;74:22-29.

7. Duy PQ, Berberoglu MA, Beattie CE, et al. Celular responses to recurrent pentylenetetrazole-induced seizures in the adult zebrafish brain. Neuroscience. 2017;349:118-127.

8. Cole TB, Robbins CA, Wenzel HJ, et al. Seizures and neuronal damage in mice lacking vesicular zinc. Epilepsy Research. 2000;39(2):153-169. 